\title{
Biomedical waste disposal systems of health facilities in Ethiopia
}

\author{
Sisay Derso ${ }^{1 *}$, Girum Taye ${ }^{2}$, Theodros Getachew ${ }^{2}$, Atkure Defar ${ }^{2}$, Habtamu Teklie$^{2}$, Kassahun Amenu², Terefe \\ Gelibo² $^{2}$ Abebe Bekele ${ }^{2}$
}

${ }^{1}$ Environmental Health Research Team, Ethiopian Public Health Institute, Addis Ababa, Ethiopia

${ }^{2}$ Health System Research Directorate, Ethiopian Public Health Institute, Addis Ababa, Ethiopia

\begin{abstract}
Background: Biomedical waste generated from health and health-related activities can be grouped as general waste and hazardous waste. This remains true if and only if there is proper on-site handling, such as the segregation and separation of waste based on the type and nature of the source.

Methods: A stratified random sampling design was used to provide representative results for Ethiopia, for various types of facility and management authorities, and for each of the 11 regions. Totally, 1327 health facilities were assessed using the World Health Organization (WHO) inventory tools.

Results: Nationally, medical waste in $32.6 \%$ of the studied health facilities was stored in covered containers, and in about $27 \%$ of them it was stored in another protected environment. About $40 \%$ of health facilities stored their medical waste in unprotected areas. Twenty-eight (2.6\%) and $420(39.3 \%)$ health facilities used 2 -chamber industrial incinerators and 1-chamber drum incinerators, respectively. About $58 \%$ of health facilities used unsafe waste treatment methods. The proportion of using safe medical waste disposal method was high in referral hospitals (87.9\%). This shows the utilization of safe medical waste disposal methods is in decreasing order from higher to lower levels of organization in health facilities.

Conclusion: The present study showed a preliminary finding on the waste disposal systems of health facilities at the national level. Dumping biomedical waste outside the health facility is common, and access to common waste facilities is limited. Therefore, a holistic approach to safe medical waste management practices, including the collection process (handling, sorting, and segregation), storage, treatment and final disposal is crucial in all types of health facilities, regardless of the level of organization, ownership, or geographic distribution.

Keywords: Health Facility, Biomedical Waste, Disposal, Incinerator, Ethiopia

Citation: Derso S, Taye G, Getachew T, Defar A, Teklie H, Amenu K, et al. Biomedical waste disposal systems of health facilities in Ethiopia. Environmental Health Engineering and Management Journal 2018; 5(1): 29-37. doi: 10.15171/EHEM.2018.05.
\end{abstract}

Article History:

Received: 3 August 2017 Accepted: 3 December 2017 ePublished: 6 January 2018

\section{Introduction}

Healthcare waste can be defined as the total waste stream that is generated from healthcare establishments, health-related research facilities, and laboratories. Hospitals, clinics, laboratories, medical research centers, pharmaceutical manufacturing plants, pharmacies, blood banks, veterinary health care centers, and home healthcare activities are some of the generators of healthcare waste irrespective of volumes, characteristics, and composition $(1,2)$.

Biomedical waste generated from health-related activities can be broadly grouped as general waste and hazardous waste. This remains true if and only if there is proper on-site handling, such as segregation, separation, and storing with a unique color-coded storage system. There are different estimates regarding the share of hazardous and non-hazardous constituents of healthcare waste. This difference was observed in a study conducted in Ethiopia. The results of a cross-sectional study conducted in selected hospitals in Addis Ababa indicated that the median waste generation rate varied between 0.361 and $0.669 \mathrm{~kg} /$ patient $/ \mathrm{d}$ and was comprised of $58.69 \%$ non-hazardous and $41.31 \%$ hazardous wastes (3). This difference was also observed in health centers in rural and urban settings. A study conducted in Addis Ababa revealed that the mean $( \pm \mathrm{SD})$ healthcare waste generation rate was $9.61 \pm 3.28 \mathrm{~kg} / \mathrm{d}$, of which (38\%) $3.64 \pm 1.45 \mathrm{~kg} / \mathrm{d}$ was general or non-hazardous waste and (62\%) $5.97 \pm 2.31$ $\mathrm{kg} / \mathrm{d}$ was hazardous (4). On the other hand, rural health centers contributed about $0.93 \pm 0.3 \mathrm{~kg} / \mathrm{d}(52.0 \%)$ general waste and $0.86 \pm 0.33 \mathrm{~kg} / \mathrm{d}$ (48.0\%) hazardous waste (5). In general, the amount of waste generated increased as 
the number of patients increased. In addition, the rate of waste generation depended on the facility type; public health facilities generated a higher proportion of total healthcare waste than private facilities (3).

Waste generated at healthcare facilities needs an adequate and appropriate management mechanism before disposal, including all activities involved in waste generation, segregation, transportation, storage, treatment, and final disposal (6). Out of the many medical waste treatment technologies, incineration is the most preferable and common treatment method for medical waste in Ethiopia and elsewhere. Incineration ranks third in the waste management hierarchy, accompanied by source reduction, reuse, recycling, and final disposal/landfilling. Incineration is an engineered process that involves thermal decomposition through thermal oxidation at elevated temperatures (usually $900^{\circ} \mathrm{C}$ and greater) to destroy the organic fraction of the waste (7). However, the inadequate or inefficient incineration of medical waste can result in high concentrations of toxic pollutants being released into the atmosphere and in bottom and fly ash, which comprises toxic organic and inorganic compounds, threatening public health as well as the environment. The Environmental Protection Agency of the United States of America found in 1994 that emissions from incinerators in healthcare facilities were responsible for high levels of chemicals such as dioxin and furan in the atmosphere (8). Next to incineration, landfills for medical waste are ranked second as a final waste management technology in the Ethiopian healthcare system. However, the use of landfills as refuse dumps is gradually expanding the problems of health hazards in the environment and among the general population. Selling biomedical waste to scavengers is another disposal method, but scavengers are always the victims of disease due to having contact with such wastes (9).

All individuals exposed to healthcare waste are at risk of being injured or infected. This includes the medical staff, inpatients and outpatients, support staff, waste handlers, waste scavengers, and the general public, particularly children who are exposed to materials from the disposed-of medical waste. However, in 2005 World Health Organization (WHO) study indicated that the rate of such injuries depends on the medical discipline. For instance, the prevalence of needlestick injury among healthcare providers in a university hospital in Germany was $46.9 \%(n=91 / 194)$ among medical staff in surgery and $18.7 \%(n=53 / 283)$ among HCWs in pediatrics. Of all occupational groups, physicians had the highest risk of experiencing needlestick injuries $(55.1 \%, \mathrm{n}=129 / 234)$ (10). In general, the medical and ancillary staff as well as sanitary experts can be injured if the waste has not been safely packed. In this respect, sharps are considered one of the most dangerous categories of waste. Many injuries occur because syringe needles or other sharps have not been collected in safety boxes or because the boxes have been overfilled. At dumpsites, scavengers may also come in contact with infectious waste during their recycling activities if the waste has not been properly disposed of or treated (11).

Biomedical waste handling is a hazardous activity that requires a high standard of training and skill. It calls for specific training that depends on the nature of the work in the service delivery unit, the hazards and possibility of worker exposure, and the responsibilities of individual workers. A study conducted in Addis Ababa showed that only $19.8 \%$ of medical waste handlers were trained to handle medical waste and none was immunized against hepatitis B virus (HBV) (12). Due to the lack of adequate training and low awareness of biomedical waste handling and management, the $\mathrm{HBV}$ was detected in $8 \%$ of healthcare waste handlers and $0.8 \%$ of non-medical waste handlers in selected hospitals in Addis Ababa and 6.0\% of healthcare waste handlers and $1.0 \%$ non-medical waste handlers in Gondar town health institutions $(12,13)$.

The number of health facilities in Ethiopia has been greatly emphasized since the implementation of the health sector development program in 1990; in this program, the expansion of health service facilities was one of the program's pillars. Due to this and other related factors, the type and quantity of disposable medical equipment and logistics are increasing and may ultimately result in a higher rate of medical waste generation in the country. In Ethiopia, as in many other developing countries, little is known about safe waste disposal and the treatment of medical waste. Furthermore, there is no comprehensive, nationally representative data on the types of health facilities (hospitals, health centers, health posts, clinics), type of administration (governmental, non-governmental, and private), region, or urbanity in Ethiopia. Therefore, the main objective of the current study was to understand the existing waste disposal system in Ethiopian health facilities and to determine key influencing factors for safe waste management practices. The study also aimed to deliver information on the release through inefficient incineration of toxic pollutants, which may increase the risk of cancer and other teratogenic effects.

\section{Methods \\ Data collection}

The data for this study was taken from the 2014 Ethiopia Service Provision Assessment Plus (ESPA+) Survey. The sample for the survey was a stratified random sample designed to provide representative results for Ethiopia, for different facility types (hospitals, health centers, health posts, clinics), different management authorities (governmental, non-governmental, private), places of residence (rural/urban), and for each of the 11 regions of the country. The data was collected by a trained health professional recruited by the Ethiopian Public Health Institute. All field staff obtained detailed training on the survey, including theory, the administration of 
questionnaires and the observational checklist, computer programming, and practical and field pilot tests. Totally, 1327 health facilities were assessed. All hospitals, selected health centers, private clinics, and health posts were assessed using a structured questionnaire and an observational checklist.

\section{Survey Instrument}

The WHO healthcare waste management inventory tools were used to assess and capture data on waste management at different departments of each health facility. A facility inventory questionnaire was used to obtain information on how the facilities dispose of and manage healthcare wastes in each of the priority services. The facility inventory questionnaire collected information on the functional status of disposal systems, type of disposal system (maintenance and management), and service delivery environment. The data collectors interviewed the most knowledgeable persons at each facility and/or service. If another provider needed to give some specific information, that provider was invited (or visited, if appropriate) and questioned.

\section{Data collection approaches}

Following the preparation of the final questionnaires in English, the questionnaires were translated into three major local languages, Amharigna, Oromiffa, and Tigrigna. Both the English and the translated inventory questionnaires were loaded onto tablet computers which were used during interviews to ask questions and record responses (Computer Assisted Personal InterviewingCAPI) in each respondent's preferred language.

\section{Sampling}

The sample size for this survey was determined by a combination of census and random samples. The Ethiopia health service system structure, organized into a 3-tier system: Level 1 (primary hospital, health center, and their satellite health posts) constituted the Primary Health Care Unit (PHCU); Level 2 was composed of general hospitals; and Level 3 was organized by teaching or specialized hospitals) was followed during sampling. The private-forprofit and NGO health sectors in Ethiopia have seen rapid expansion in recent years, and due to their importance, they were considered in sampling.

In general, the sample of this survey included all hospitals and a sample of health centers and private facilities. Health posts were selected independently. Due to their number and service, all hospitals were included in the survey, allowing for the inclusion of newly identified hospitals. A representative sample of health centers and clinics was selected for the survey. A total sample size of 1327 health facilities was selected, including 321 health posts and 10 newly identified hospitals.

Data analysis
The chi-square test was used to analyze bivariate data, and the logistic regression method was used for univariate and multivariate analysis. The medical waste treatment methods used in each health facility were considered as an outcome variable by categorizing them into safe medical waste disposal methods and unsafe medical waste disposal methods.

The independent variables for this analysis were facility type, managing authority, region, urban/rural, and the availability of waste disposal guidelines. Bivariate analysis revealed that, at a $5 \%$ level of significance, all independent variables (potential factors) had an association with medical waste disposal methods (safe/unsafe). Therefore, all independent variables were considered in both univariate and multivariate logistic regression models to identify the potential predictors of using safe medical waste disposal methods. The unit of analysis for this study was all health facilities covered in the SPA project. Both bivariate and multivariate statistical analyses were carried out to identify the potential determinants of using safe medical waste disposal methods in health facilities using SPSS version 20 software.

\section{Hypothesis}

To test the hypothesis, the following categorization gave the outcome variable:

- If the health facilities used Incinerator (2-chamber industrial), Incinerator (1-chamber drum/brick), open burning (pit or protected), dump no burning (covered pit or pit latrine), dump no burning (protected ground or pit), removal offsite (stored in covered container), or removal offsite (stored in other protected environment) and burned in other facility, they were recorded as using safe medical waste disposal methods.

- If the health facilities used open burning (flat ground no protection), dump no burning (flat ground, no protection), dump no burning (open pit no protection), removal off-site (stored unprotected) or barrel (Bermel), they were recorded as using unsafe medical waste disposal methods.

The independent variables considered were facility type, managing authority, region, urban/rural, and waste disposal guideline availability.

\section{Results}

Descriptive analysis

After medical waste is generated during the provision of healthcare services at health facilities, it needs to be handled and stored in a safe place to prevent nosocomial infections and to protect the public. Nationally, 32.6\% of the health facilities stored medical waste in a covered container, and about $27 \%$ of health facilities stored it in another protected environment. The remaining $40 \%$ of health facilities stored their medical waste in unprotected areas, like an open field or barrel, and did not store waste 
completely, as shown in Table 1.

Table 2 presents the existing waste treatment methods in the studied health facilities. Twenty-eight $(2.6 \%)$ and $420(39.3 \%)$ health facilities used 2-chamber industrial incinerators and 1-chamber drum incinerators, respectively. The remaining $58 \%$ of health facilities (the majority) used unsafe waste treatment methods, including open burning and dumping in unsanitary ways.

\section{Bivariate analysis}

Table 3 presents the status of using safe medical waste disposal method in selected health facilities by background characteristics. At a $1 \%$ level of significance, the results indicate the status of using safe medical waste disposal methods is significantly associated with facility type, managing authority, region, urban/rural, and availability of guidelines for waste management in the service area. The use of safe medical waste disposal methods was high in referral hospitals (87.9\%) followed by general hospitals (86.6\%), health centers (79.5\%), higher clinics (78.6\%), primary hospitals (76.8\%), medium clinics (64.3\%), health posts $(52.6 \%)$, and lower clinics (51\%). This shows that the utilization of safe medical waste disposal methods has a generally decreasing trend with the decreasing level of health facilities from the higher to the lower tier of the Ethiopian health service structure.

Among the 1327 studied health facilities, 28 (87.5\%) health facilities managed under the NGO authority

Table 1. Percentage of facilities by medical waste storage methods used

\begin{tabular}{lc}
\hline Methods used for medical waste storage & No. (\%) \\
\hline Storing in covered container & $31(32.6)$ \\
Storing in another protected environment & $26(27.4)$ \\
Unprotected storage & $13(13.7)$ \\
Barrel & $8(8.4)$ \\
Stored in other facility & $3(3.2)$ \\
Other & $14(14.7)$ \\
Total & $95(100)$ \\
\hline
\end{tabular}

Table 2. Percentage of facilities by medical waste treatment methods used

\begin{tabular}{ll}
\hline Methods used for medical waste treatment & No. (\%) \\
Burn-in incinerator, 2 chambers industrial & $28(2.6)$ \\
Burn-in incinerator, 1 chamber drum & $420(39.3)$ \\
Open burning, flat ground-no protection & $208(19.4)$ \\
Open burning, pit or protected ground & $346(32.3)$ \\
Dump no burning, flat ground, no protection & $20(1.9)$ \\
Dump no burning, covered pit or pit latrine & $13(1.2)$ \\
Dump no burning, open pit, no protection & $19(1.8)$ \\
Dump no burning, protected ground or pit & $16(1.5)$ \\
Total & $1070(100)$ \\
\hline
\end{tabular}

and $527(68.6 \%)$ of the health facilities managed under government or public authority used safe medical waste disposal methods.

The use of safe medical waste disposal methods was high among facilities in Addis Ababa (106; 82.8\%) followed by Tigray (95; 72\%), S.N.N.P (130; 71\%), Dire Dawa (58; $69 \%)$, Afar (53; 67.9\%), Amhara (121; 65.8\%), Harari (37; 60.7\%), Somalia (51; 60.7\%), Gambella (48; 60\%), Oromiya (140; 59.3\%), and Benshangul (44; 57.1\%).

The proportion of health facilities using safe medical waste disposal methods was higher in urban areas ( $n=465$; $74.5 \%)$ compared with health facilities in rural areas $(\mathrm{n}=$ $418 ; 61.1 \%)$. In addition, the proportion of health facilities using safe medical waste disposal methods was high among those who had guidelines for waste management in the service area $(n=243 ; 82.7 \%)$ compared with those who did not $(n=640 ; 73.5 \%)$.

\section{Multivariate analysis}

Table 4 presents the adjusted and unadjusted odds ratios with 95\% confidence intervals which were obtained from multivariate and univariate logistic regression models by considering the variables with the status of using safe medical waste disposal methods as an outcome variable. The results of univariate logistic regression at a $5 \%$ level of significance showed that facility type, managing authority, region, urban/rural, and guidelines availability were all statistically significant in determining whether the medical waste disposal methods used were safe or not. Therefore, all of the above background characteristics should be considered as explanatory variables in the multivariate logistic regression model.

The odds ratios shown in Table 4 were used to compare the probability of an event for two groups. In this study, it helps to test the following hypotheses.

Ho: There is no association between medical waste disposal methods in health facilities and background characteristics ( $P$ value $>0.05)$.

H1: There is an association between medical waste disposal methods in health facilities and background characteristics $(P$ value $<0.05)$.

From Table 4, it can be seen that some $P$ values for medical waste disposal methods by region had a less than $5 \%$ level of significance and its corresponding 95\% CI for the odds ratio (OR) does not include one, implies somehow, there is an association between the two variables with the following interpretation.

The odds of using a safe medical waste disposal method in the assessed health facilities were 3.345 times greater among health facilities in the Dire Dawa region than facilities in the Tigray region.

At a 5\% level of significance, almost all $P$ values in measuring the association between medical waste disposal methods in the assessed health facilities and background characteristics were less than 0.05 , and its corresponding 95\% CI for OR does not include one, it indicates that there 
Table 3. Status of using safe medical waste disposal methods in health facilities by background characteristics

\begin{tabular}{|c|c|c|c|c|}
\hline \multirow{2}{*}{ Background Characteristics } & \multicolumn{2}{|c|}{ Medical waste disposal methods } & \multirow{2}{*}{ Chi-square } & \multirow{2}{*}{$P$ value } \\
\hline & Safe, No. (\%) & Unsafe, No. (\%) & & \\
\hline Facility type (\%) & & & 115.626 & $<0.01$ \\
\hline Referral hospital (2.5) & $29(87.9)$ & $4(12.1)$ & & \\
\hline General hospital (10.1) & $116(86.6)$ & $18(13.4)$ & & \\
\hline Primary hospital (4.2) & $43(76.8)$ & $13(23.2)$ & & \\
\hline Health centre (22.4) & $237(79.5)$ & $61(20.5)$ & & \\
\hline Health post (24.2) & $169(52.6)$ & $152(47.4)$ & & \\
\hline Higher clinic (5.3) & $55(78.6)$ & $15(21.4)$ & & \\
\hline Medium clinic (12.7) & $108(64.3)$ & $60(35.7)$ & & \\
\hline Lower clinic (18.6) & $126(51)$ & $121(49)$ & & \\
\hline Managing authority & & & 12.269 & 0.007 \\
\hline Government or public (57.9) & $527(68.6)$ & $241(31.4)$ & & \\
\hline Other governmental (0.9) & $7(58.3)$ & $5(41.7)$ & & \\
\hline Private for profit (38.8) & $321(62.3)$ & $194(37.7)$ & & \\
\hline NGO (2.4) & $28(87.5)$ & $4(12.5)$ & & \\
\hline Region & & & 31.334 & 0.001 \\
\hline Tigray $(9,4)$ & $95(72)$ & $37(28)$ & & \\
\hline Afar (5.9) & $53(67.9)$ & $25(32.1)$ & & \\
\hline Amhara (13.9) & $121(65.8)$ & $63(34.2)$ & & \\
\hline Oromiya (17.8) & $140(59.3)$ & $96(40.7)$ & & \\
\hline Somalia (6.3) & $51(60.7)$ & $33(39.3)$ & & \\
\hline Benshangul (5.8) & $44(57.1)$ & $33(42.9)$ & & \\
\hline S.N.N.P (13.8) & $130(71)$ & $53(29)$ & & \\
\hline Gambella (6.0) & $48(60)$ & $32(40)$ & & \\
\hline Harari (4.6) & $37(60.7)$ & $24(39.3)$ & & \\
\hline Addis Ababa (9.4) & $106(82.8)$ & $22(17.2)$ & & \\
\hline Dire Dawa (6.3) & $58(69)$ & $26(31)$ & & \\
\hline Urban/Rural & & & 64.684 & $<0.01$ \\
\hline Urban (47.7) & $465(74.5)$ & $159(25.5)$ & & \\
\hline Rural (52.3) & $418(61.1)$ & $266(38.9)$ & & \\
\hline Guideline availability & & & 10.084 & 0.001 \\
\hline Yes (25.2) & $243(82.7)$ & $51(17.3)$ & & \\
\hline No (74.8) & $640(73.5)$ & $231(26.5)$ & & \\
\hline
\end{tabular}

is an association between the two variables.

Moreover, the results can be interpreted as showing that among the assessed health facilities, general hospitals are 7.537 times more likely to use safe medical waste disposal methods than referral hospitals. In addition, primary hospitals, health centers, health posts, and medium clinics are 5.914, 4.335, 4.639, and 8.227 times more likely, respectively, to use safe medical waste disposal methods compared to a referral hospital.

At a $5 \%$ level of significance, there was no association between medical waste disposal methods in health facilities and facility type, managing authority, availability of guidelines for waste management in the service area, or urban/rural background characteristics.

\section{Discussion}

Biomedical waste generated from health and healthrelated activities can be broadly grouped as general waste and hazardous waste. This remains true if and only if there is proper on-site handling, segregation, and separation of waste with regard to the type and nature of the source. The present study revealed that about $33 \%$ of health facilities use covered containers, and $27 \%$ of them store their medical waste in protected environments. Conversely, about $40 \%$ of the studied health facilities stored their waste in an unprotected environment (Table 1). Waste produced in healthcare facilities in developing countries has raised serious concerns because of inappropriate treatment and final disposal practices, which can impose negative impacts on public health and the environment (14). Having this concept in mind, the present healthcare waste storage practices in Ethiopian health facilities could threaten the public as well as the environment. In addition, the unimproved waste storage practices of health facilities could lead to insufficient waste incineration and complicated final disposal of medical waste.

In general, poor medical waste handling and storage practices potentially create risks to health workers, clients, the environment, and human health. One study conducted in Ethiopia indicated that due to the lack of adequate waste handling and biomedical waste storage practices, the HBV was detected in waste handlers ( $8 \%$ of healthcare waste handlers and $0.8 \%$ in non-medical waste handlers) in selected hospitals in Addis Ababa and 6.0\% 
Table 4. Adjusted odds ratios with $95 \%$ confidence intervals for using safe medical waste disposal methods in health facilities

\begin{tabular}{|c|c|c|c|}
\hline Background Characteristics & Adjusted OR & $95 \% \mathrm{Cl}$ for adjusted OR & $P$ value \\
\hline \multicolumn{4}{|l|}{ Facility type } \\
\hline Referral Hospital & 1 & & \\
\hline General Hospital & 7.537 & $(1.297,43.807)$ & 0.024 \\
\hline Primary Hospital & 5.914 & $(1.782,19.626)$ & 0.004 \\
\hline Health Centre & 4.335 & $(1.097,17.134)$ & 0.036 \\
\hline Health post & 4.639 & $(1.299,16.567)$ & 0.018 \\
\hline Higher clinic & 1.617 & $(0.453,5.771)$ & 0.459 \\
\hline Medium clinic & 8.227 & $(1.888,35.846)$ & 0.005 \\
\hline Lower clinic & 1.767 & $(0.977,3.194)$ & 0.060 \\
\hline \multicolumn{4}{|l|}{ Managing authority } \\
\hline Government or public & 1 & & \\
\hline Other governmental & 0.236 & $(0.048,1.151)$ & 0.074 \\
\hline Private for profit & 0.294 & $(0.044,1.954)$ & 0.205 \\
\hline NGO & 0.596 & $(0.167,2.131)$ & 0.426 \\
\hline \multicolumn{4}{|l|}{ Region } \\
\hline Tigray & 1 & & \\
\hline Afar & 1.398 & $(0.683,2.861)$ & 0.360 \\
\hline Amhara & 1.932 & $(0.822,4.541)$ & 0.131 \\
\hline Oromiya & 0.938 & $(0.492,1.787)$ & 0.845 \\
\hline Somalia & 0.671 & $(0.361,1.247)$ & 0.207 \\
\hline Benshangul & 1.024 & $(0.46,2.278)$ & 0.954 \\
\hline S.N.N.P & 0.964 & $(0.456,2.039)$ & 0.923 \\
\hline Gambella & 1.233 & $(0.642,2.368)$ & 0.530 \\
\hline Harari & 1.198 & $(0.558,2.572)$ & 0.643 \\
\hline Addis Ababa & 0.698 & $(0.318,1.530)$ & 0.369 \\
\hline Diredawa & 3.345 & $(1.142,9.798)$ & 0.028 \\
\hline \multicolumn{4}{|l|}{ Urban/Rural } \\
\hline Urban & 1 & & \\
\hline Rural & 1.23 & $(0.82,1.843)$ & 0.317 \\
\hline \multicolumn{4}{|l|}{ Guideline availability } \\
\hline Yes & 1 & & \\
\hline No & 1.078 & $(0.704,1.651)$ & 0.704 \\
\hline
\end{tabular}

of healthcare waste handlers and $1 . \%$ non-medical waste handlers in Gondar town health institutions $(12,13)$. Such findings are in agreement with the WHO estimation that injections with contaminated syringes caused 21 million HBV infections (32\% of all new infections), 2 million hepatitis C virus (HCV) infections $(40 \%$ of all new infections), and $260000 \mathrm{HIV}$ infections (5\% of all new infections) globally (15).

Furthermore, the improper medical waste handling and storage practices of the majority of Ethiopian health facilities could result in needlestick and sharp object injuries. All individuals exposed to healthcare waste are at risk of being injured or infected. This includes medical staffs, inpatients and outpatients, support staff, waste handlers, scavengers, and the public, particularly children or scavengers who are exposed to materials from disposedof medical waste. However, the study showed that the rate of such injuries depends on the medical discipline; for instance, the prevalence of needlestick injury among healthcare providers in a university hospital in Germany was $46.9 \%(n=91 / 194)$ among medical staff in surgery and $18.7 \%(n=53 / 283)$ among healthcare workers in pediatrics. Of all occupational groups, physicians have the highest risk of experiencing needlestick injuries (55.1\%; n $=129 / 234)(10)$.
Incineration is the most preferable and common treatment method for medical waste in Ethiopia and elsewhere. Incineration ranks third in the waste management hierarchy, accompanied by source reduction, reuse, recycling, and final disposal/landfilling. About $94 \%$ of health facilities in the present study used burning as their preferred treatment method for medical waste; $3 \%$ used 2-chamber incinerators, $39 \%$ used 1-chamber incinerators, and the remaining health facilities, a substantial number, used open burning as their medical waste treatment (Table 2). Inadequate or inefficient incineration of medical waste can result in the release of high concentrations of toxic pollutants into the atmosphere and to bottom and fly ash, which comprises toxic organic and inorganic compounds. These toxic releases comprise organic emissions such as polychlorinated dibenzo-dioxins/furnace (PCDD/Fs) and polycyclic aromatic hydrocarbons (PAHs), inorganic emissions, and ashes containing toxic metals. These products are carcinogenic and affect the development, reproduction, and immune systems of humans (7). Historically, health concerns raised by incineration focused on communities living near the incinerator. Recently, the WHO identified three potentially exposed populations: the local population through inhalation, workers at the facility, and the larger regional population 
through the food chain and bioaccumulate pollutants (16). Furthermore, incinerators dangerously pollute technologies that will virtually undermine the objectives of the treaty on persistent organic pollutants (POPs). The Stockholm Convention on POPs, which was ratified by Ethiopia (2003) and entered into action (2004), identifies waste incinerators as the principal source of dioxins and furans, which are among the initial 12 substances being targeted for continuing minimization and ultimate elimination by the global community (17). Considering this, many countries have taken action to treat and dispose of health facility waste using environmentally safer, noncombustion technologies. Ethiopia, as a global entity, could follow this approach as well.

In this study, the result of bivariate analysis shown in Table 3 indicates that at a $1 \%$ level of significance, the status of using safe medical waste disposal method was significantly associated with facility type, managing authority, region, urban/rural, and availability of guidelines for waste management in the service area. This finding is in agreement with a study conducted in India which revealed that biomedical waste management practices were better among hospital staff in comparison with private medical practitioners and marginally higher among those in urban areas in comparison with those in rural areas (18).

The management of healthcare waste is an integral part of a national healthcare system. The waste management system consists of all activities related to the handling, treatment, disposal, or recycling of waste materials. The purpose of a waste management system is to make sure that waste materials are removed from the source or location where they are generated and treated, disposed of, or recycled in a safe and proper manner (19). However, in this study, the multivariate logistic regression analysis revealed that general hospitals were 7.537 times more likely to use safe medical waste disposal methods than referral hospitals. Furthermore, primary hospitals, health centers, health posts, and medium clinics were 5.914, 4.335, 4.639, and 8.227 times more likely, respectively, to use safe medical waste disposal methods than referral hospitals (Table 4). As a result, a holistic approach to healthcare waste management including a clear delineation of responsibilities, occupational health and safety programs, waste minimization and segregation, the development and adoption of safe and environmentally sound technologies, and capacity building should be considered in all types of health facilities in both rural and urban settings.

Education and awareness of waste and waste management are increasingly important from a global perspective of resource management. The role of sustainable waste management is to reduce the amount of waste that is discharged into the environment by reducing the amount of waste generated. In Ethiopia, medical waste is mostly dumped in the open field or burned in open fires, which may contribute to air pollution and the pollution of other environmental compartments, including groundwater sources and soil due to leachate and recharging. To improve such situations, strategies must be designed for all types of health facilities with special emphasis on those using less safe waste management methods, regardless of facility type, location, or managing authority. Waste pollutes the environment and threatens human health; therefore, tackling such problems requires rigorous and continual public health education. Educating personnel will also improve the efficiency of waste management systems and minimize the possible health and environmental risks (19).

This study also showed that the availability of healthcare waste management guidelines contributes to safe waste handling and management (Figure 1). The current results indicated that more health facilities with waste management guidelines are practicing safe waste management than health facilities without guidelines. The results further illustrated that 231 health facilities that did not have guidelines during the survey managed their medical waste in an unsafe manner in a higher proportion than those with guidelines ( 51 health facilities only). Another study conducted in Ethiopia, which is in agreement with the current findings, indicated that the management of healthcare waste requires well-organized documents, including policy documents, regulations, and technical guidelines, but the existing healthcare

Guideline Availability

$\square$ Yes $(25,2) \quad$ No $(74.8)$

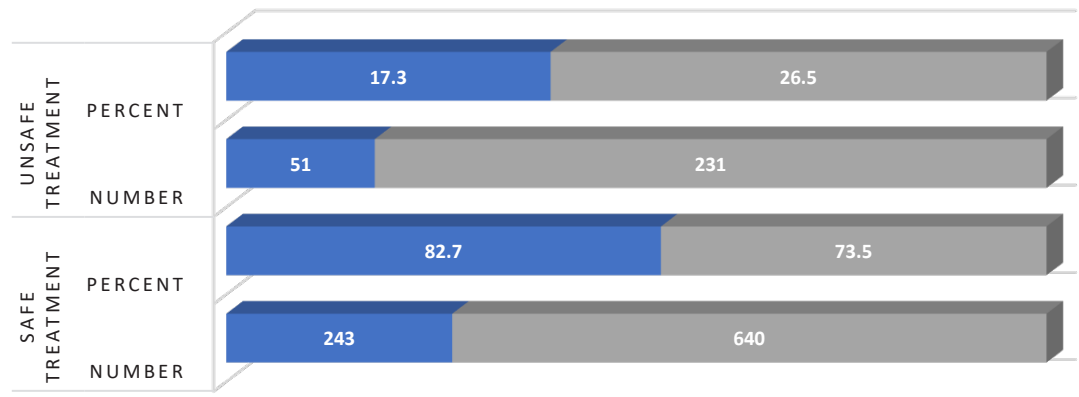

Figure 1. Availability of guidelines in health facility and its contribution to waste management. 
waste management practices do not obey the principles of waste management in general. In addition, mandate overlapping and limited cooperation between various stakeholders and responsible sectors were identified as major barriers to safe medical waste management (20). Some aspects of healthcare waste management can be improved by providing training for medical staff, waste handlers, support staff, and the public on proper waste management and source reduction. Another step toward improvement is the preparation of wholesome legislation and policy documents on healthcare waste management with a clear description of the mandates to different interested parties. However, significant financial and infrastructure challenges remain. These include the costs for special containers, human resources, administrative and regulatory capacities, as well as disposal systems and technologies.

\section{Conclusion}

The present study showed a preliminary finding on the waste disposal systems of health facilities at a national level. The results revealed that $60 \%$ of health facilities stored waste in a protected environment, and the remaining $40 \%$ stored it in an insanitary way. About $94 \%$ of the studied facilities used burning medical waste as a major treatment method, among which only about $42 \%$ used a standard incinerator; the remaining facilities practiced open burning.

Utilization of safe medical waste disposal methods is significantly associated with facility type, managing authority, region, urban/rural, and availability of guidelines for waste management in the service area. This study showed that the utilization of safe medical waste disposal methods had a decreasing trend with the decreasing levels of organization of the health facilities. At a 5\% level of significance, the bivariate analysis showed that all background characteristics considered in this study (facility type, managing authority, region, urban/ rural, and guideline availability) were associated with the respective medical waste disposal methods in the selected health facilities. Based on the multivariate analysis, however, it seems that, at a 5\% level of significance, general hospitals are more likely to use safe medical waste disposal methods than referral hospitals.

Dumping biomedical waste outside the health facility is a common practice, and access to common waste facilities is still limited. Therefore, a holistic approach to safe medical waste management practices, including the collection process (handling, sorting, and segregation), storage, treatment, and the final disposal is crucial in all types of health facility, regardless of their level of organization, ownership, or geographic distribution. Furthermore, surveillance, monitoring, and continuous awareness-raising tasks should be considered to improve waste management systems in all health facilities across the country.

\section{Acknowledgments}

The authors would like to acknowledge the Ethiopian Public Health Institute for its financial and logistic support of this research paper.

\section{Ethical issues}

The authors hereby certify that all data collected during the study under the section of waste disposal is as stated in this manuscript, and no data from the section has been or will be published elsewhere separately.

\section{Competing interests}

The authors have declared that they have no conflicts of interest.

\section{Authors' contributions}

SD performed the overall manuscript writing. GT helped with data analysis and application of software. TG participated in data collection and the overall coordination of the survey. AD, HT, KA, and TG participated in data collection. $\mathrm{AB}$ guided and managed the data collection and manuscript preparation.

\section{References}

1. Khajuria A, Kumar A. Assessment of Healthcare Waste Generated by Government Hospital in Agra City, India. Our Nature 2007; 5(1): 25-30. doi: 10.3126/on.v5i1.794.

2. Pruss A, Giroult E, Rushbrook P. Safe Management of Wastes from Health-care Activities. Geneva: World Health Organization; 1999.

3. Debere MK, Gelaye KA, Alamdo AG, Trifa ZM. Assessment of the health care waste generation rates and its management system in hospitals of Addis Ababa, Ethiopia, 2011. BMC Public Health 2013; 13: 28. doi: 10.1186/1471-2458-13-28.

4. Tadesse ML, Kumie A. Healthcare waste generation and management practice in government health centers of Addis Ababa, Ethiopia. BMC Public Health 2014; 14: 1221. doi: 10.1186/1471-2458-14-1221.

5. Azage M, Kumie A. Healthcare waste generation and its management system: the case of health centers in West Gojjam Zone, Amhara Region, Ethiopia. Ethiop J Health Dev 2010; 24(2):119-26. doi: 10.4314/ejhd. v24i2.62960.

6. Odette RH, Masika J, Venance T, Soatiana JE, Christiane NA, Lamine CM, Bin L. Assessment of healthcare waste generation and its management systems: a prevalence survey of the healthcare facilities in Madagascar. J Environ Sci Toxicol Food Technol 2014; 8: 20-9.

7. Singh S, Prakash V. Toxic environmental releases from medical waste incineration: a review. Environ Monit Assess 2007; 132(1-3): 67-81. doi: 10.1007/ s10661-006-9503-3.

8. Malkan S, Nelson J. Global trends in responsible 
healthcare waste management-A perspective from health Care without harm. Waste Manag 2005; 25(6): 570-2.

9. Hussein H, Musah BA, Guo E. Disposal of health facility waste in landfill sites and its effects on the people of Gbalahi community in the Tamale metropolis. Int Res J Public Environ Health 2014; 1(1): 1-9.

10. Wicker S, Jung J, Allwinn R, Gottschalk R, Rabenau HF. Prevalence and prevention of needlestick injuries among health care workers in a German university hospital. Int Arch Occup Environ Health 2008; 81(3): 347-54. doi: 10.1007/s00420-007-0219-7.

11. Manual G. Preparation of national healthcare waste management plans in sub-Saharan countries. Geneva: World Health Organization; 2005.

12. Shiferaw $Y$, Abebe T, Mihret A. Hepatitis B virus infection among medical waste handlers in Addis Ababa, Ethiopia. BMC Res Notes 2011; 4(1): 479. doi: 10.1186/1756-0500-4-479.

13. Anagaw B, Shiferaw Y, Anagaw B, Belyhun Y, Erku W, Biadgelegn F, et al. Seroprevalence of hepatitis B and $\mathrm{C}$ viruses among medical waste handlers at Gondar town Health institutions, Northwest Ethiopia. BMC Res Notes 2012; 5: 55. doi: 10.1186/1756-0500-5-55.
14. Diaz LF, Savage GM, Eggerth LL. Alternatives for the treatment and disposal of healthcare wastes in developing countries. Waste Manag 2005; 25(6): 62637. doi: 10.1016/j.wasman.2005.01.005.

15. World Health Organazation. WHO core principles for achieving safe and sustainable management of health-care waste. Geneva, Switzerland: WHO; 2007.

16. Batterman S, Water S. Findings on an Assessment of Small-scale Incinerators for Health-care Waste. Geneva: WHO; 2004.

17. Convention S. Stockholm convention on persistent organic pollutants. UNEP; 2001.

18. Rao PH. Report: Hospital waste management-awareness and practices: a study of three states in India. Waste Manag Res 2008; 26(3): 297-303. doi: $10.1177 / 0734242 \times 08088693$.

19. Demirbas A. Waste management, waste resource facilities and waste conversion processes. Energy Convers Manag 2011; 52(2): 1280-7. doi: 10.1016/j. enconman.2010.09.025.

20. Haylamicheal ID, Desalegne SA. A review of legal framework applicable for the management of healthcare waste and current management practices in Ethiopia. Waste Manag Res 2012; 30(6): 607-18. doi: $10.1177 / 0734242 \times 11419891$. 